\title{
Troxerutin Protects Against Myocardial Ischemia/Reperfusion Injury Via Pi3k/Akt Pathway in Rats
}

\author{
Liliang Shu ${ }^{a} \quad$ Wanzhe Zhang ${ }^{b} \quad$ Chen Huang ${ }^{a}$ Gongcheng Huang ${ }^{a} \quad$ Gang Su$^{a}$ \\ aDepartment of Cardiovascular Surgery, The First Affiliated Hospital of Zhengzhou University, \\ Zhengzhou, ${ }^{b}$ Department of Nephrology, The Second Affiliated Hospital of Zhengzhou University, \\ Zhengzhou, China
}

\section{Key Words}

Myocardial ischemia/reperfusion injury • Troxerutin • PI3K/Akt pathway

\begin{abstract}
Background/Aims: Troxerutin, also known as vitamin P4, has been commonly used in the treatment of chronic venous insufficiency (CVI) disease. However, its effect on in vivo myocardial ischemia/reperfusion (I/R) injury, a model that closely mimics acute myocardial infarction in humans, is still unknown. Methods: The myocardial I/R injury rat model was created with troxerutin preconditioning. Myocardial infarct size was evaluated by the Evans blue-TTC method. Hemodynamic parameters, including the heart rate (HR), left ventricular end-diastolic pressure (LVEDP), left ventricular systolic pressure (LVSP), maximal rate of rise in blood pressure in the ventricular chamber $(+\mathrm{dp} / \mathrm{dt}$ max), and maximal rate of decline in blood pressure in the ventricular chamber (-dp/dt max) were monitored. Serum TNF- $\alpha$ and IL-10 were determined by ELISA kit. Cell apoptosis was detected by MTT method. Results: Troxerutin preconditioning significantly reduced myocardial infarct size, improved cardiac function, and decreased the levels of creatine kinase (CK), aspartate aminotransferase (AST) and lactate dehydrogenase (LDH) in the I/R injury rat model. The serum and mRNA levels of TNF- $\alpha$ and IL-10 as well as some apoptosis markers (Bax, Caspase 3 ) also decreased. Moreover, troxerutin pretreatment markedly increased the phosphorylation of Akt, and blocking PI3K activity by LY294002 abolished the protective effect of troxerutin on I/R injury. Conclusion: Troxerutin preconditioning protected against myocardial I/R injury via the PI3K/Akt pathway.
\end{abstract}

\section{Introduction}

(C) 2017 The Author(s) Published by S. Karger AG, Basel

Ischemic heart disease secondary to acute myocardial infarction is a severe health problem in the world, which is the leading cause of death worldwide. Although timely reperfusion is necessary to limit the cell death resulting from cardiac ischemia, sudden 
restoration of blood flow to ischemic myocardium may exaggerate the myocardial injury. This phenomenon is known as myocardial ischemia/reperfusion (I/R) injury [1]. Some factors contribute to the pathological process of myocardial I/R injury, such as oxidative stress [2], autophagy [3], calcium overload [4], inflammation [5], and mitochondrial dysfunction [6].

The PI3K/Akt pathway plays a crucial role in cell growth and cell survival. The activated Akt pathway can regulate cell survival through phosphorylation of a variety of downstream targets, such as pro-apoptotic protein, transcription factors and endothelial nitric oxide synthase (eNOS)[7-9]. The activation of the PI3K/Akt pathway protects the brain from cerebral I/R injury by preventing neuronal apoptosis [10]. Activation of the PI3K/Akt pathway also protects against myocardial I/R injury by decreasing oxidative stress, repressing the inflammatory cascade, and inhibiting apoptosis in vivo and in vitro [11-14].

Troxerutin, also known as vitamin P4, is natural flavonoid rutin present in tea, coffee, cereal grains and a variety of fruits and vegetables. It has various biological functions, including improving capillary function, reducing capillary fragility and abnormal leakage, and anti-erythrocytic and anti-thrombotic effects [15]. Troxerutin has protective effects in several tissues, including kidney [16], liver [17] and brain [18]. In the context of myocardial $\mathrm{I} / \mathrm{R}$ injury, troxerutin has a protective effect against reperfusion injury of diabetic myocardium by attenuating GSK-3 $\beta$ activity and subsequently reducing apoptosis [19]. The $\mathrm{PI} 3 \mathrm{~K} /$ Akt pathway is important in mediating the effect of troxerutin [20]. However, there is no report on a troxerutin preconditioning effect on myocardial I/R injury in healthy bodies or the role of the PI3K/Akt pathway in troxerutin's effects in vivo.

In this study, we identified the protective properties of troxerutin in a myocardial $\mathrm{I} / \mathrm{R}$ injury rat. Our findings provide in vivo and in vitro evidence that the cardioprotective effects of troxerutin are mediated through PI3K/Akt signaling pathways.

\section{Materials and Methods}

\section{Animals}

Male Wistar rats (40 rats, weighing 250-300 g) were supplied by the medical animal center of our institute. The animals were fed a standard rodent chow diet with free access to water and housed in a temperature-controlled $\left(25 \pm 3^{\circ} \mathrm{C}\right)$, pathogen-free room with a 12-hour light, 12-hour dark cycle. The procedures were performed in accordance with the Guide for the Care and Use of Laboratory Animals published by the US National Institutes of Health (NIH Publication no. 80-23, revised 1996) and approved by the local animal care committee.

\section{Experimental group}

After acclimation to their environment for 7 days, all rats were randomly allocated into four groups (n=10/group): 1) sham group; 2) I/R group; 3) I/R+Troxerutin group; 4) I/R+Troxirutin+LY294002 group. For those undergoing I/R surgery, the rats were administered with troxerutin ( $150 \mathrm{mg} / \mathrm{kg}$ ) by oral gavage or LY294002 (an inhibitor of PI3K, $0.2 \mathrm{mg} / \mathrm{kg}$ ) by subcutaneous injection (S.C.) for 4 weeks before IR operation. The sham group was given saline by oral gavage for 4 weeks before I/R operation.

\section{In vivo myocardial $I / R$ model}

The rats were anesthetized with isoflurane inhalation. A left thoracotomy was performed to expose the beating heart. The I/R animal model was established by ligation of the left anterior descending (LAD) coronary artery for $30 \mathrm{~min}$ followed by reperfusion for $45 \mathrm{~min}$ (by reopening of LAD) [21]. The chest was closed in layers, and the animals were allowed to recover. The successful performance of coronary occlusion and reperfusion was verified by an immediate fall in coronary flow at the onset of index ischemia and its recovery upon reperfusion. 


\section{Cellular Physiology Cell Physiol Biochem 2017;44:1939-1948 \begin{tabular}{l|l|l} 
and Biochemistry & DOI: 10.1159/000485884 & $\begin{array}{l}\text { (c) } 2017 \text { The Author(s). Published by S. Karger AG, Basel } \\
\text { www.karger.com/cpb }\end{array}$
\end{tabular}}

Shu et al.: Troxerutin Protects Against Myocardial I/R Injury

\section{Determination of myocardial infarct size}

Myocardial infarct size was evaluated by the Evans blue-TTC method as previously described [22]. Briefly, the ligature around the left anterior descending coronary artery was reoccluded after reperfusion. Four milliliters of $1 \%$ Evans blue dye was injected into the vena cava to track the perfused region and the area at risk (AAR). The myocardial AAR area was identified as the region lacking blue staining. The ventricles of the hearts were sliced transversely into 2 -mm-thick slices and incubated in $1 \%$ tetrazolium chloride solution (TTC) for 20 min to identify the infarct area. The weights of the infarct area (white), AAR (red), and nonischemic zones of the LV (blue) were measured. Infarct size is reported as a percentage of the AAR mass.

\section{Hemodynamic examination}

Hemodynamic examination was performed as previously described [22]. The right carotid artery was exposed via a neck dissection. Hemodynamic parameters, including the heart rate (HR), left ventricular end-diastolic pressure (LVEDP), left ventricular systolic pressure (LVSP), maximal rate of rise in blood pressure in the ventricular chamber ( $+\mathrm{dp} / \mathrm{dt}$ max), and maximal rate of decline in blood pressure in the ventricular chamber (-dp/dt max), were measured and recorded.

\section{Serum parameters measurement}

Serum CK and LDH were measured in the clinical laboratory of our institution according to the manufacturer's protocols. The results are reported as U/L. Serum TNF- $\alpha$ and IL-10 were determined by an ELISA kit following the manufacturer's instructions.

\section{Cell culture}

The H9c2 cells, a rat cardiomyoblast line, were purchased from American Type Culture Collection (Manassas, VA, USA). The cell lines had been passaged for less than 6 months when used for experiments. H9c2 cells were maintained in DMEM containing $4.5 \mathrm{~g} / \mathrm{L}$ glucose with Glutamax from Invitrogen (Basel, Switzerland) and supplemented with 10\% FBS, $5 \mathrm{mmol} / \mathrm{L}$ HEPES, $1 \mathrm{mmol} / \mathrm{L}$ sodium pyruvate and 500 $\mu \mathrm{g} / \mathrm{ml}$ penicillin-streptomycin. Cells were kept at $37^{\circ} \mathrm{C}$ in a humidified atmosphere of $5 \% \mathrm{CO}_{2}$ in air.

Other standard methods

RNA isolation, qPCR, MTT Assay and Western blot (WB) were performed as described previously [23$26]$. For WB, equal amounts of proteins from five mouse livers in each group ( $n=5$ /group) were pooled, and single or duplicate loading was used.

\section{Statistical Analysis}

All data are presented as the mean \pm SEM. Statistical analysis was carried out using Student's $t$ test between two groups and one-way ANOVA between multiple groups. In all cases, values of $\mathrm{P}<0.05$ were considered statistically significant.

\section{Results}

Troxerutin preconditioning decreases the infarct size induced by I/R operation

As shown in Fig. 1A, compared with the sham group, AAR area was increased dramatically by I/R operation, while there was no significant difference between the three groups with I/R operation. The infarct size induced by I/R operation was decreased in the $\mathrm{I} / \mathrm{R}+$ troxerutin group $(\mathrm{P}<0.05)$, while the effect of troxerutin on infarct size was blocked by LY294002 (Fig. 1B).

Further, we detected the serum levels of several myocardial infarction markers. As shown in Table 1, similar to the results of infarct size, I/R significantly increased cTnI, $\mathrm{CK}$ and $\mathrm{LDH}$. Pre-treatment with troxerutin (I/R+troxerutin) significantly reduced these markers compared with the I/R group $(\mathrm{P}<0.05)$, which effect was also blocked by LY294002. 
Fig. 1. Troxerutin preconditioning decreases the infarct size induced by $I / R$ operation. (A) AAR area and (B) infarct size were evaluated in all the groups. Group 1, sham group; group 2, I/R group; Group 3, I/ $\mathrm{R}+$ troxerutin group; Group 4, I/R+troxerutin+LY294002 group. Data are shown as Mean \pm SEM. ${ }^{*} \mathrm{P}<0.05$ vs I/R group.

Fig. 2. Effect of troxerutin on hemodynamics of I/R injury rat. The hemodynamic parameters LVSP, LVEDP, +dp/dt max and -dp/dt max were measured. Group 1, sham group; group 2, I/R group; Group 3, I/ $\mathrm{R}+$ troxerutin group; Group 4, I/R+troxerutin+LY294002 group. Data are shown as Mean \pm SEM. * $\mathrm{P}<0.05$ vs sham group; \# $\mathrm{P}<0.05$ vs I/R group. \& $\mathrm{P}<0.05$ vs I/ $\mathrm{R}+$ troxerutin group.
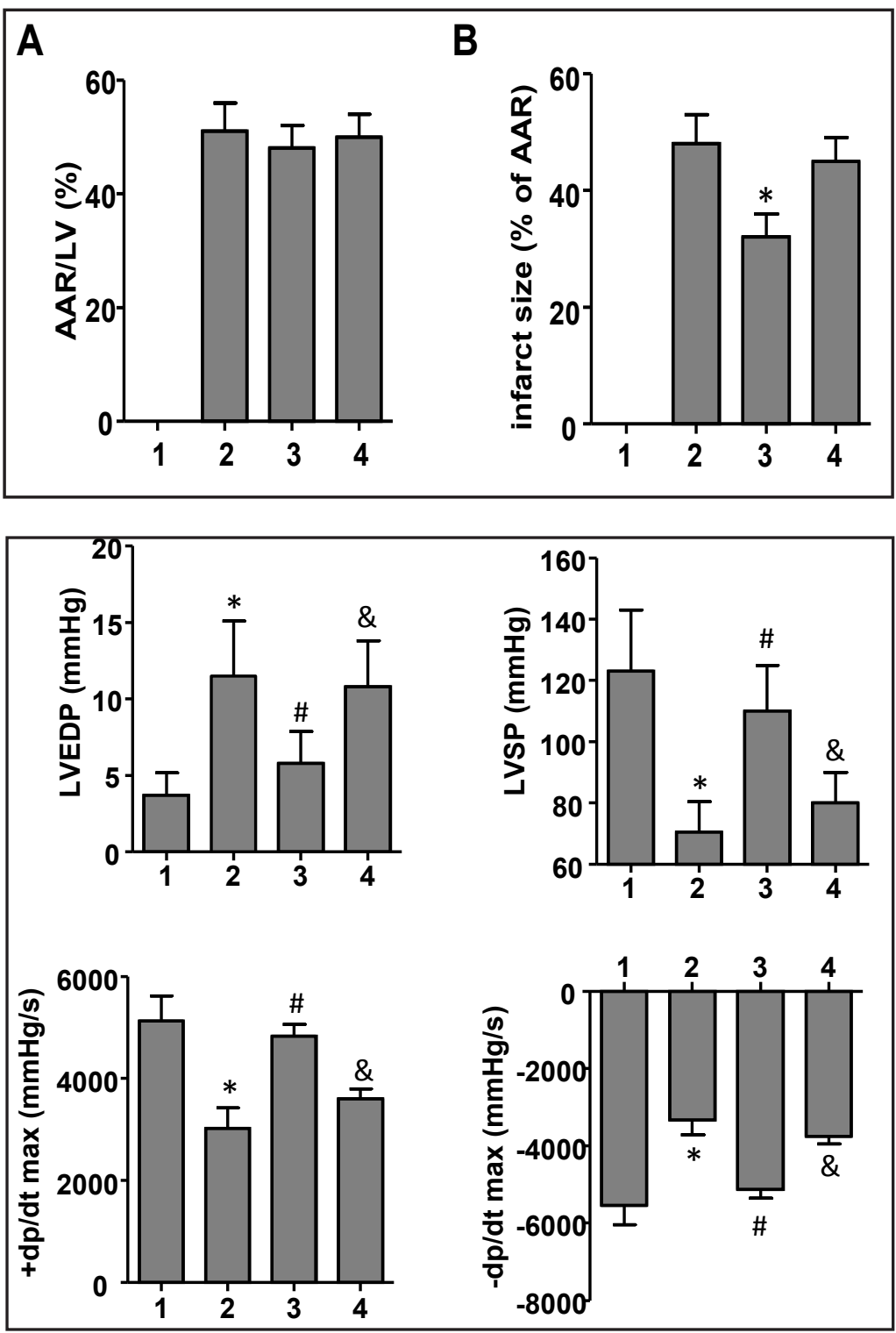

Table 1. Effect of troxerutin preconditioning on serum cTnI, CK and LDH in myocardial $\mathrm{I} / \mathrm{R}$ injury rat model. a, $\mathrm{P}<0.01$ vs sham group; $\mathrm{b}, \mathrm{P}<0.01$ vs $\mathrm{I} / \mathrm{R}$ group; $\mathrm{c}, \mathrm{P}<0.01$ vs $\mathrm{I} / \mathrm{R}+$ troxerutin group

\begin{tabular}{llll}
\hline Group & $\mathrm{cTnI}(\mathrm{ng} / \mathrm{ml})$ & $\mathrm{CK}(\mathrm{U} / \mathrm{L})$ & LDH $(\mathrm{U} / \mathrm{L})$ \\
\hline Sham & $0.24 \pm 0.03$ & $1293.51 \pm 124.75$ & $1075.28 \pm 122.35$ \\
$\mathrm{I} / \mathrm{R}$ & $20.81 \pm 2.43 \mathrm{a}$ & $5236.24 \pm 157.63 \mathrm{a}$ & $3284.02 \pm 326.93 \mathrm{a}$ \\
$\mathrm{I} / \mathrm{R}+$ troxerutin & $10.74 \pm 1.79 \mathrm{ab}$ & $2702.46 \pm 195.27 \mathrm{ab}$ & $1268.51 \pm 147.28 \mathrm{ab}$ \\
I/R+troxerutin+LY294002 & $18.75 \pm 2.23 \mathrm{ac}$ & $4638.92 \pm 268.35 \mathrm{ac}$ & $3027.86 \pm 203.17 \mathrm{ac}$ \\
\hline
\end{tabular}

Table 2. Effect of troxerutin preconditioning on myocardial apoptosis rate in myocardial I/R injury rat model. a, $\mathrm{P}<0.01$ vs sham group; $\mathrm{b}, \mathrm{P}<0.01$ vs $\mathrm{I} / \mathrm{R}$ group; c, $\mathrm{P}<0.01$ vs $\mathrm{I} / \mathrm{R}+$ troxerutin group

\begin{tabular}{ll}
\hline Group & Myocardial apoptosis rate (\%) \\
\hline Sham & $2.87 \pm 0.32$ \\
I/R & $31.25 \pm 2.76^{\mathrm{a}}$ \\
$\mathrm{I} / \mathrm{R}+$ troxerutin & $18.43 \pm 2.53^{\mathrm{ab}}$ \\
$\mathrm{I} / \mathrm{R}+$ troxerutin+LY294002 & $25.32 \pm 3.56^{\mathrm{ac}}$ \\
\hline
\end{tabular}


Fig. 3. Effect of troxerutin on I/R induced myocardial apoptosis. (A) the mRNA and (B) protein levels of apoptosis markers (BAX, caspase 3 and BCL-2). (C) Caspase 3 activity. Group 1, sham group; group 2, I/R group; Group 3, I/R +troxerutin group; Group 4, I/ $\mathrm{R}+$ troxerutin +LY294002 group. Data are shown as Mean \pm SEM. ${ }^{*} \mathrm{P}<0.05$, ${ }^{* *} \mathrm{P}<0.01$ vs sham group; \# $\mathrm{P}<0.05$ \#\# $\mathrm{P}<0.01$ vs I/R group. \& $P<0.05$ \&\& $\mathrm{P}<0.01$ vs $\mathrm{I} / \mathrm{R}+$ troxerutin group.

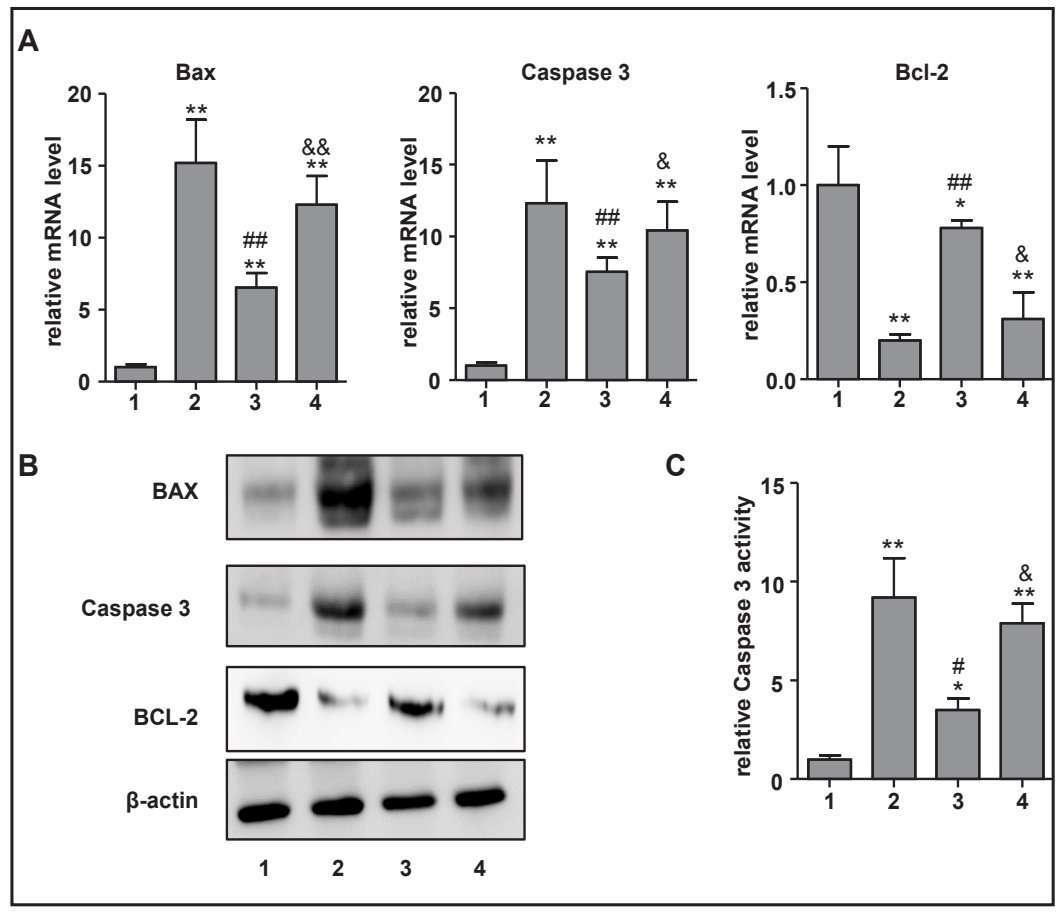

Effect of troxerutin on hemodynamics of $I / R$ injury rat

As shown in Fig. 2, the levels of LVSP, + dp/dtmax and -dp/dtmax were significantly decreased, whereas LVEDP was significantly increased, in the I/R group compared with the sham group. Troxerutin preconditioning significantly increased LVSP, $+\mathrm{dp} / \mathrm{dtmax}$ and -dp/dtmax, whereas troxerutin preconditioning reduced LVEDP in the I/R+troxerutin group compared to the I/R group. However, the effects of troxerutin on hemodynamics were significantly reversed in the I/R+troxerutin+LY294002 group compared to the IR+troxerutin group.

\section{Effect of troxerutin on I/R-induced myocardial apoptosis}

We also detected the myocardial apoptosis in this study. As shown in Table 2, the myocardium apoptosis rate in the I/R group was significantly increased compared with the sham group. Troxerutin preconditioning significantly decreased the myocardium apoptosis rate in the I/R+troxerutin group compared to the I/R group. However, the decreased myocardium apoptosis rate caused by troxerutin was significantly reversed in the I/ $\mathrm{R}+$ troxerutin+LY294002 group.

We also measured several apoptosis markers in myocardial tissues. As shown in Fig. $3 \mathrm{~A} \& \mathrm{~B}$, compared with the sham group, the mRNA and protein levels of two apoptosis markers (Bax and Caspase 3) were increased and the anti-apoptosis marker (Bcl-2) was reduced in the I/R group. Troxerutin preconditioning significantly attenuated the increases in Bax and Caspase 3 and the decrease in Bcl-2 induced by I/R, which was reversed in the $\mathrm{I} / \mathrm{R}+$ troxerutin+LY294002 group. We also detected the Caspase 3 activity in these groups, which showed a similar pattern with its mRNA expression (Fig. 3C).

Effect of troxerutin preconditioning on serum cytokine levels and heart ICAM-1 expression

As shown in Fig. 4, serum levels of CRP, TNF- $\alpha$, IL-1 $\beta$ and IL-10 as well as the mRNA level of ICAM-1, the indicator of neutrophil-endothelial cell interactions, were significantly increased in the I/R group over the sham group. These cytokines were significantly decreased in troxerutin-preconditioned rats compared with I/R rats. However, their levels were significantly increased in rats treated with troxerutin and LY29400 compared to troxerutin alone. 


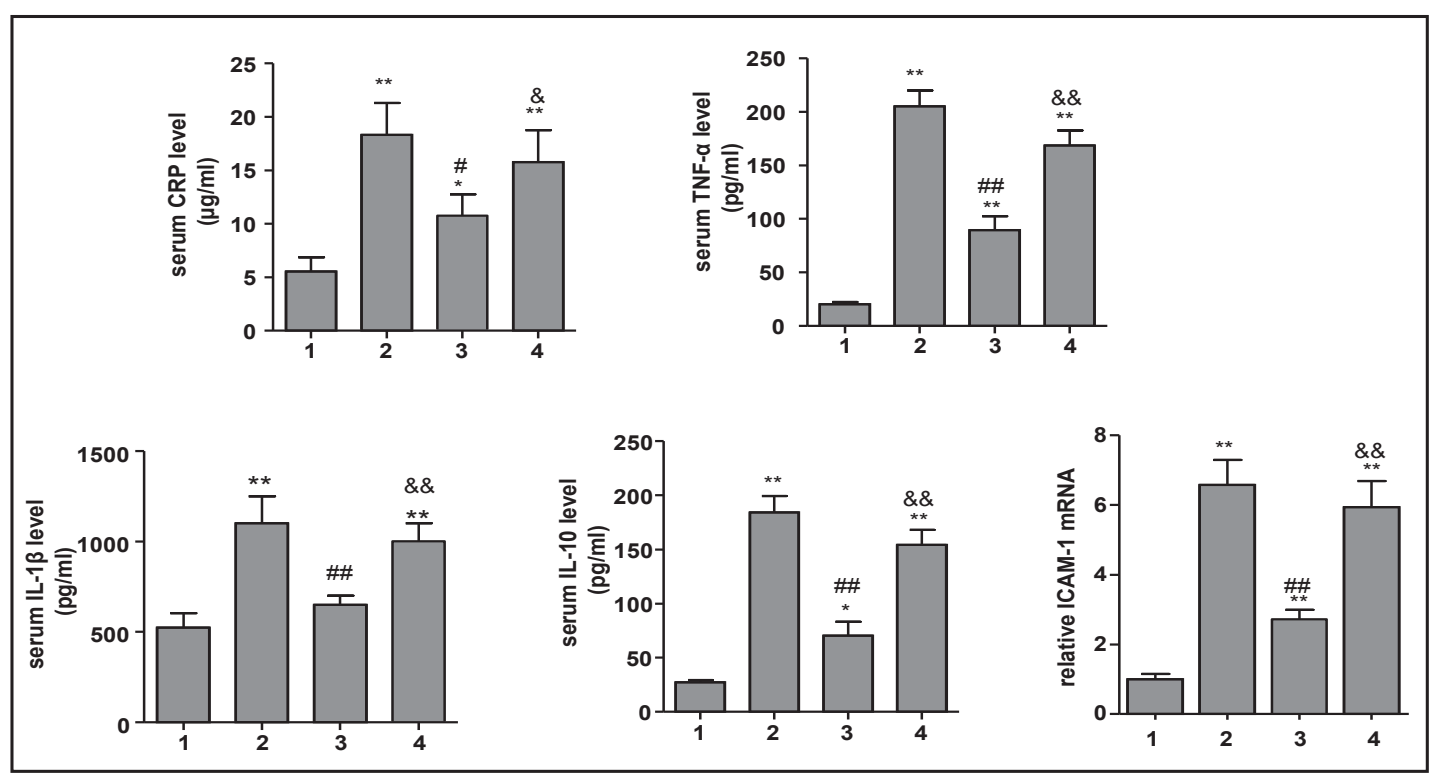

Fig. 4. Effect of troxerutin preconditioning on serum cytokine levels and heart ICAM-1 expression. Serum CRP, TNF- $\alpha$, IL-10 and IL-1 $\beta$ as well as heart ICAM-1 mRNA expression were measured. Group 1, sham group; group 2, I/R group; Group 3, I/R+troxerutin group; Group 4, I/R+troxerutin +LY294002 group. Data are shown as Mean \pm SEM. * $\mathrm{P}<0.05$, ** $\mathrm{P}<0.01$ vs sham group; \# $\mathrm{P}<0.05 \# \# \mathrm{P}<0.01$ vs I/R group. $\& \mathrm{P}<0.05$ \&\& $\mathrm{P}<0.01$ vs I/R+troxerutin group.

Fig. 5. Protective effect of troxerutin against I/R Injury is mediated via PI3K/Akt pathway. (A) The p-Akt and total Akt protein levels in I/R rat model with troxerutin and LY294002 treatment. The p-Akt and total Akt protein levels in $\mathrm{H} 9 \mathrm{c} 2$ cells treated with troxerutin for different (B) doses and (C) times. (D) The cell viability of $\mathrm{H} 9 \mathrm{c} 2$ cells detected by MTT assay with $\mathrm{H}_{2} \mathrm{O}_{2}$ and troxerutin treatment. Group 1 , sham group; group 2, I/R group; Group 3, I/ $\mathrm{R}+$ troxerutin group; Group 4, I/R+troxerutin+LY294002 group. Data are shown as Mean \pm SEM. ${ }^{* *} \mathrm{P}<0.01$ vs con; \#\# $\mathrm{P}<0.01$ vs $\mathrm{H}_{2} \mathrm{O}_{2}$ treatment.
A

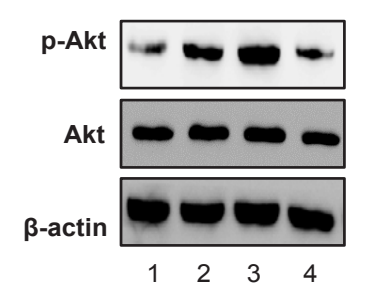

C

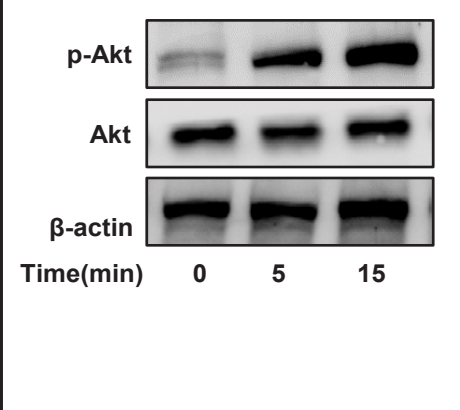

B
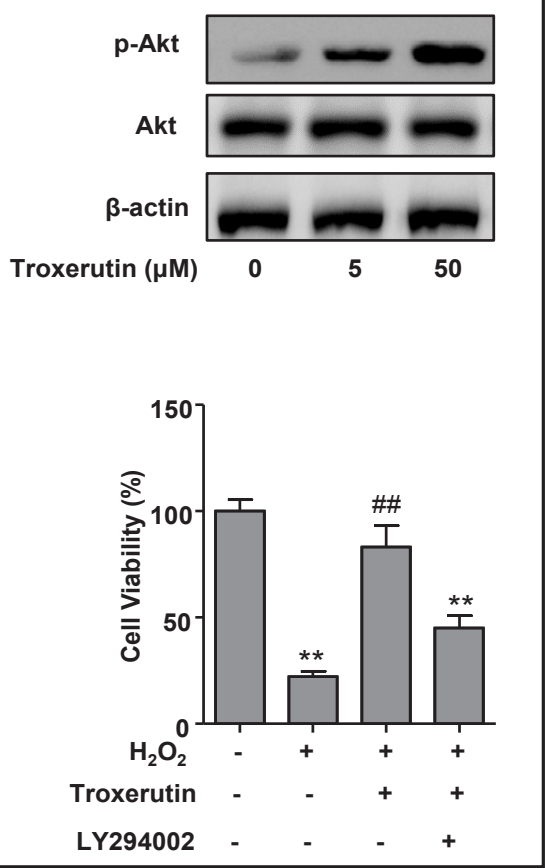

Protective effect of troxerutin against I/R Injury is mediated via PI3K/Akt pathway

The activation of the PI3K/Akt pathway has a protective effect against I/R injury, and troxerutin can activate the PI3K/Akt pathway. As shown in Fig. 5A, the t-Akt level had no 


\section{Cellular Physiology Cell Physiol Biochem 2017;44:1939-1948 \\ \begin{tabular}{l|l} 
DOI: 10.1159/000485884 & $\begin{array}{l}\text { O 2017 The Author(s). Published by S. Karger AG, Basel } \\
\text { www.karger.com/cpb }\end{array}$
\end{tabular} \\ Shu et al.: Troxerutin Protects Against Myocardial I/R Injury}

significant change between these groups, while p-Akt increased moderately in the I/R groups and increased more in the I/R+troxerutin group. The I/R+troxerutin + LY29400 group showed significantly lower $\mathrm{p}$-Akt than the I/R+troxerutin group.

We also studied the effect of troxerutin in vitro. As shown in Fig. 5B, troxerutin significantly increased p-Akt in H9c2 cells in a dose- and time-dependent manner. ROS release is thought to be important in the pathogenesis of myocardial I/R injury [27]. Our study found that the treatment of $\mathrm{H} 9 \mathrm{c} 2$ cells with troxerutin markedly reduced $\mathrm{H}_{2} \mathrm{O}_{2}$-induced cell death compared to $\mathrm{H}_{2} \mathrm{O}_{2}$ treatment alone. In addition, inhibition of the Akt pathway by LY294002 completely blocked the protective ability of troxerutin in $\mathrm{H}_{2} \mathrm{O}_{2}$-treated $\mathrm{H} 9 \mathrm{c} 2$ cells (Fig. 5C). These results strongly suggest that Akt pathway activation is necessary for a protective effect of troxerutin in myocardial I/R injury.

\section{Discussion}

In the present study, preconditioning with troxerutin significantly ameliorated the myocardial I/R injury, including reduction of myocardial infarct size, decreased inflammatory cytokines, and inhibited cardiomyocyte apoptosis. Mechanistically, our results show that the protective effect of troxerutin on myocardial I/R injury is mediated by the PI3K/Akt pathway.

Flavonoids are a subgroup of the more extended family of polyphenols. More than 5000 flavonoids have been identified, each with a basic structure containing two benzene rings with a pyrane ring in the middle [28]. A number of flavonoid compounds have cardioprotective effects against I/R injury, such as tilianin [29], vitexin [30], icariin [22] and quercetin [11]. Here, we reported the protective effect of troxerutin on myocardial I/R injury, which is a naturally occurring flavonoid.

The inflammatory response is stimulated by I/R injury, leading to the release of inflammatory cytokines, which are critical mediators of infarct healing and cardiac remodeling [31]. In addition, inhibiting inflammation can decrease myocardial I/R injury and preserve cardiac function $[32,33]$. In the present study, we found that preconditioning of troxerutin reduced the production of inflammatory cytokines induced by I/R injury.

Cardiomyocyte apoptosis is dominant in the pathogenesis of myocardial I/R injury [34] and is responsible for a significant amount of cardiomyocyte death in I/R injury [35, 36]. Suppressing cell apoptosis attenuates cardiomyocyte loss and prevents I/R injury [37-40]. In the present study, the cardiomyocyte apoptotic rate decreased, and myocardial infarction size was reduced in the I/R+troxerutin group compared with the I/R group. We also found that troxerutin treatment reduced $\mathrm{H}_{2} \mathrm{O}_{2}$-induced cell apoptosis in vitro. These results suggest that the protective effect of troxerutin might be related to a reduction of cellular apoptosis.

Cardiomyocyte necrosis is another type of cell death in myocardial I/R injury [34]. LDH is a stable cytosolic enzyme present in mammalian cells that is released when cell necrosis occurs [41]. In our study, we found that I/R induced serum LDH dramatically, while troxerutin preconditioning reduced $\mathrm{LDH}$, which suggested that troxerutin might prevent myocardial I/R injury by also reducing cell necrosis.

Troxerutin also has been reported to block the endoplasmic reticulum stress and oxidative stress pathway in brain and liver of mice $[18,42,43]$, and troxerutin could increase the activity of anti-oxidant enzymes and limiting the lipid peroxidation [44]. We need to do further study to explore if these pathways also participate the protective effect of troxerutin in myocardial I/R injury.

\section{Conclusion}

In summary, our study revealed the protective effect of troxerutin preconditioning on myocardial I/R injury, which was mediated by the PI3K/Akt pathway. Our results provide new evidence for the clinical use of troxerutin in ischemic heart diseases. 


\section{Cellular Physiology Cell Physiol Biochem 2017;44:1939-1948 \begin{tabular}{l|l|l} 
and Biochemistry Published onlIne: December 08, 2017 & $\begin{array}{l}\text { (c) } 2017 \text { The Author(s). Published by S. Karger AG, Basel } \\
\text { www.karger.com/cpb }\end{array}$
\end{tabular}}

Shu et al.: Troxerutin Protects Against Myocardial I/R Injury

\section{Disclosure Statement}

The authors declare that there is no financial conflict of interest.

\section{References}

1 Kambe M, Bessho R, Fujii M, Ochi M, Shimizu K: Sivelestat reduces myocardial ischemia and reperfusion injury in rat hearts even when administered after onset of myocardial ischemia. Interact Cardiovasc Thorac Surg 2009;8:629-634.

-2 Tao L, Gao E, Jiao X, Yuan Y, Li S, Christopher TA, Lopez BL, Koch W, Chan L, Goldstein BJ, Ma XL: Adiponectin cardioprotection after myocardial ischemia/reperfusion involves the reduction of oxidative/ nitrative stress. Circulation 2007;115:1408-1416.

3 Zhu T, Yao Q, Wang W, Yao H, Chao J: iNOS Induces Vascular Endothelial Cell Migration and Apoptosis Via Autophagy in Ischemia/Reperfusion Injury. Cell Physiol Biochem 2016;38:1575-1588.

4 Murata M, Akao M, O’Rourke B, Marban E: Mitochondrial ATP-sensitive potassium channels attenuate matrix $\mathrm{Ca}(2+)$ overload during simulated ischemia and reperfusion: possible mechanism of cardioprotection. Circ Res 2001;89:891-898.

5 Oyama J, Blais C, Jr., Liu X, Pu M, Kobzik L, Kelly RA, Bourcier T: Reduced myocardial ischemia-reperfusion injury in toll-like receptor 4-deficient mice. Circulation 2004;109:784-789.

6 Lesnefsky EJ, Moghaddas S, Tandler B, Kerner J, Hoppel CL: Mitochondrial dysfunction in cardiac disease: ischemia--reperfusion, aging, and heart failure. J Mol Cell Cardiol 2001;33:1065-1089.

7 Franke TF, Hornik CP, Segev L, Shostak GA, Sugimoto C: PI3K/Akt and apoptosis: size matters. Oncogene 2003;22:8983-8998.

-8 Ou HC, Lee WJ, Lee SD, Huang CY, Chiu TH, Tsai KL, Hsu WC, Sheu WH: Ellagic acid protects endothelial cells from oxidized low-density lipoprotein-induced apoptosis by modulating the PI3K/Akt/eNOS pathway. Toxicol Appl Pharmacol 2010;248:134-143.

-9 Wang HB, Yang J, Ding JW, Chen LH, Li S, Liu XW, Yang CJ, Fan ZX: RNAi-Mediated Down-Regulation of CD47 Protects against Ischemia/Reperfusion-Induced Myocardial Damage via Activation of eNOS in a Rat Model. Cell Physiol Biochem 2016;40:1163-1174.

10 Hua F, Ma J, Ha T, Kelley J, Williams DL, Kao RL, Kalbfleisch JH, Browder IW, Li C: Preconditioning with a TLR2 specific ligand increases resistance to cerebral ischemia/reperfusion injury. J Neuroimmunol 2008;199:75-82.

11 Liu H, Guo X, Chu Y, Lu S: Heart protective effects and mechanism of quercetin preconditioning on antimyocardial ischemia reperfusion (IR) injuries in rats. Gene 2014;545:149-155.

12 Li X, Hu X, Wang J, Xu W, Yi C, Ma R, Jiang H: Short-Term Hesperidin Pretreatment Attenuates Rat Myocardial Ischemia/Reperfusion Injury by Inhibiting High Mobility Group Box 1 Protein Expression via the PI3K/Akt Pathway. Cell Physiol Biochem 2016;39:1850-1862.

13 Yu H, Zhang H, Zhao W, Guo L, Li X, Li Y, Zhang X, Sun Y: Gypenoside Protects against Myocardial IschemiaReperfusion Injury by Inhibiting Cardiomyocytes Apoptosis via Inhibition of CHOP Pathway and Activation of PI3K/Akt Pathway In vivo and In vitro. Cell Physiol Biochem 2016;39:123-136.

14 Wu H, Ye M, Yang J, Ding J, Dong W, Wang X: Nicorandil Protects the Heart from Ischemia/Reperfusion Injury by Attenuating Endoplasmic Reticulum Response-induced Apoptosis Through PI3K/Akt Signaling Pathway. Cell Physiol Biochem 2015;35:2320-2332.

15 Maurya DK, Balakrishnan S, Salvi VP, Nair CK: Protection of cellular DNA from gamma-radiation-induced damages and enhancement in DNA repair by troxerutin. Mol Cell Biochem 2005;280:57-68.

16 Fan SH, Zhang ZF, Zheng YL, Lu J, Wu DM, Shan Q, Hu B, Wang YY: Troxerutin protects the mouse kidney from d-galactose-caused injury through anti-inflammation and anti-oxidation. Int Immunopharmacol 2009;9:91-96.

17 Zhang ZF, Fan SH, Zheng YL, Lu J, Wu DM, Shan Q, Hu B: Troxerutin protects the mouse liver against oxidative stress-mediated injury induced by D-galactose. J Agric Food Chem 2009;57:7731-7736. 


\section{Cellular Physiology Cell Physiol Biochem 2017;44:1939-1948 \begin{tabular}{l|l|l} 
and Biochemistry Published onlIne: December 08, 2017 & $\begin{array}{l}\text { (c) } 2017 \text { The Author(s). Published by S. Karger AG, Basel } \\
\text { www.karger.com/cpb }\end{array}$
\end{tabular}}

18 Lu J, Wu DM, Hu B, Cheng W, Zheng YL, Zhang ZF, Ye Q, Fan SH, Shan Q, Wang YJ: Chronic administration of troxerutin protects mouse brain against D-galactose-induced impairment of cholinergic system. Neurobiol Learn Mem 2010;93:157-164.

19 Mokhtari B, Badalzadeh R, Alihemmati A, Mohammadi M: Phosphorylation of GSK-3beta and reduction of apoptosis as targets of troxerutin effect on reperfusion injury of diabetic myocardium. Eur J Pharmacol 2015;765:316-321.

20 Lu J, Wu DM, Hu B, Zheng YL, Zhang ZF, Wang YJ: NGF-Dependent activation of TrkA pathway: A mechanism for the neuroprotective effect of troxerutin in D-galactose-treated mice. Brain Pathol 2010;20:952-965.

21 Badalzadeh R, Mohammadi M, Najafi M, Ahmadiasl N, Farajnia S, Ebrahimi H: The additive effects of ischemic postconditioning and cyclosporine-A on nitric oxide activity and functions of diabetic myocardium injured by ischemia/reperfusion. J Cardiovasc Pharmacol Ther 2012;17:181-189.

22 Meng X, Pei H, Lan C: Icariin Exerts Protective Effect Against Myocardial Ischemia/Reperfusion Injury in Rats. Cell Biochem Biophys 2015;73:229-235.

23 Lee SM, Zhang Y, Tsuchiya H, Smalling R, Jetten AM, Wang L: Small heterodimer partner/neuronal PAS domain protein 2 axis regulates the oscillation of liver lipid metabolism. Hepatology 2015;61:497-505.

24 Tsuchiya H, da Costa KA, Lee S, Renga B, Jaeschke H, Yang Z, Orena SJ, Goedken MJ, Zhang Y, Kong B, Lebofsky M, Rudraiah S, Smalling R, Guo G, Fiorucci S, Zeisel SH, Wang L: Interactions Between Nuclear Receptor SHP and FOXA1 Maintain Oscillatory Homocysteine Homeostasis in Mice. Gastroenterology 2015;148:1012-1023 e1014.

-25 Lua I, James D, Wang J, Wang KS, Asahina K: Mesodermal mesenchymal cells give rise to myofibroblasts, but not epithelial cells, in mouse liver injury. Hepatology 2014;60:311-322.

-26 Yoon SM, Gerasimidou D, Kuwahara R, Hytiroglou P, Yoo JE, Park YN, Theise ND: Epithelial cell adhesion molecule (EpCAM) marks hepatocytes newly derived from stem/progenitor cells in humans. Hepatology 2011;53:964-973.

27 Hu X, Ma R, Lu J, Zhang K, Xu W, Jiang H, Da Y: IL-23 Promotes Myocardial I/R Injury by Increasing the Inflammatory Responses and Oxidative Stress Reactions. Cell Physiol Biochem 2016;38:2163-2172.

28 Ross JA, Kasum CM: Dietary flavonoids: bioavailability, metabolic effects, and safety. Annu Rev Nutr 2002;22:19-34.

29 Guo X, Cao W, Yao J, Yuan Y, Hong Y, Wang X, Xing J: Cardioprotective effects of tilianin in rat myocardial ischemia-reperfusion injury. Mol Med Rep 2015;11:2227-2233.

-30 Dong LY, Li S, Zhen YL, Wang YN, Shao X, Luo ZG: Cardioprotection of vitexin on myocardial ischemia/ reperfusion injury in rat via regulating inflammatory cytokines and MAPK pathway. Am J Chin Med 2013;41:1251-1266.

31 Vinten-Johansen J, Jiang R, Reeves JG, Mykytenko J, Deneve J, Jobe LJ: Inflammation, proinflammatory mediators and myocardial ischemia-reperfusion Injury. Hematol Oncol Clin North Am 2007;21:123-145.

-32 Heusch G: Cardioprotection: chances and challenges of its translation to the clinic. Lancet 2013;381:166175.

-33 Jian J, Xuan F, Qin F, Huang R: The Antioxidant, Anti-Inflammatory and Anti-Apoptotic Activities of the Bauhinia Championii Flavone are Connected with Protection Against Myocardial Ischemia/Reperfusion Injury. Cell Physiol Biochem 2016;38:1365-1375.

34 Oerlemans MI, Koudstaal S, Chamuleau SA, de Kleijn DP, Doevendans PA, Sluijter JP: Targeting cell death in the reperfused heart: pharmacological approaches for cardioprotection. Int J Cardiol 2013;165:410-422.

-35 Yang X, Qin Y, Shao S, Yu Y, Zhang C, Dong H, Lv G, Dong S: MicroRNA-214 Inhibits Left Ventricular Remodeling in an Acute Myocardial Infarction Rat Model by Suppressing Cellular Apoptosis via the Phosphatase and Tensin Homolog (PTEN). Int Heart J 2016;57:247-250.

36 Wang X, Cao Y, Shen M, Wang B, Zhang W, Liu Y, He X, Wang L, Xia Y, Ding M, Xu X, Ren J: DIDS reduces ischemia/reperfusion-induced myocardial injury in rats. Cell Physiol Biochem 2015;35:676-688.

37 Hojo Y, Saito T, Kondo H: Role of apoptosis in left ventricular remodeling after acute myocardial infarction. J Cardiol 2012;60:91-92.

-38 Zhu J, Yao K, Wang Q, Guo J, Shi H, Ma L, Liu H, Gao W, Zou Y, Ge J: Ischemic Postconditioning-Regulated miR-499 Protects the Rat Heart Against Ischemia/Reperfusion Injury by Inhibiting Apoptosis through PDCD4. Cell Physiol Biochem 2016;39:2364-2380.

39 Zhu T, Yao Q, Hu X, Chen C, Yao H, Chao J: The Role of MCPIP1 in Ischemia/Reperfusion Injury-Induced HUVEC Migration and Apoptosis. Cell Physiol Biochem 2015;37:577-591. 


\section{Cellular Physiology Cell Physiol Biochem 2017;44:1939-1948 \begin{tabular}{l|l|l} 
DOI: 10.1159/000485884 & $\begin{array}{l}\text { O 2017 The Author(s). Published by S. Karger AG, Basel } \\
\text { www.karger.com/cpb }\end{array}$ \\
\hline
\end{tabular}}

Shu et al.: Troxerutin Protects Against Myocardial I/R Injury

40 Yang J, Guo X, Ding JW, Li S, Yang R, Fan ZX, Yang CJ: RP105 Protects Against Apoptosis in Ischemia/ Reperfusion-Induced Myocardial Damage in Rats by Suppressing TLR4-Mediated Signaling Pathways. Cell Physiol Biochem 2015;36:2137-2148.

41 Parhamifar L, Andersen H, Moghimi SM: Lactate dehydrogenase assay for assessment of polycation cytotoxicity. Methods Mol Biol 2013;948:13-22.

42 Lu J, Wu DM, Zheng YL, Hu B, Cheng W, Zhang ZF, Li MQ: Troxerutin counteracts domoic acid-induced memory deficits in mice by inhibiting CCAAT/enhancer binding protein beta-mediated inflammatory response and oxidative stress. J Immunol 2013;190:3466-3479.

-43 Zhang ZF, Zhang YQ, Fan SH, Zhuang J, Zheng YL, Lu J, Wu DM, Shan Q, Hu B: Troxerutin protects against 2, 2',4, 4'-tetrabromodiphenyl ether (BDE-47)-induced liver inflammation by attenuating oxidative stressmediated NAD(+)-depletion. J Hazard Mater 2015;283:98-109.

44 Badalzadeh R, Layeghzadeh N, Alihemmati A, Mohammadi M: Beneficial effect of troxerutin on diabetesinduced vascular damages in rat aorta: histopathological alterations and antioxidation mechanism. Int J Endocrinol Metab 2015;13:e25969. 